\title{
Fernkälteversorgung zur Vermeidung von Grundwasser- erwärmungen und Nutzungskonflikten am Beispiel der Stadt Linz - Bewertung auf Basis ÖWAV-Regelblatt 207 und qualitativer Nutzwertanalyse
}

\author{
Stefan Krakow · Daniela Fuchs-Hanusch
}

Online publiziert: 4. Juli 2016

(C) Der/die Autor(en) 2016 . Dieser Artikel ist eine Open-Access-Publikation.

\begin{abstract}
Zusammenfassung Im Zuge von Untersuchungen zur Grundwasserbewirtschaftung Linz wurden im Stadtgebiet Linz erhöhte Grundwassertemperaturen festgestellt. Um einen allfälligen $\mathrm{Zu}$ sammenhang zwischen Grundwassernutzungsanlagen zur Kälteerzeugung und den erhöhten Grundwassertemperaturen zu ermitteln, wurden im Rahmen der hier vorgestellten Studie die Auswirkungen vorhandener Grundwassernutzer anhand bewilligter Konsens-

unterschieden. Die Variantenbewertung erfolgte mittels qualitativer Nutzwertanalyse. Neben ökologischen und ökonomischen Kriterien wurden in die Nutzwertanalyse auch rechtlich/soziale Kriterien einbezogen. In diesem Artikel werden neben der Wärmefahnenberechnung vorhandener Kältenutzer zwei der sieben entwickelten Fernkälteversorgungsvarianten und die Ergebnisse der Nutzwertanalyse im Detail vorgestellt.
\end{abstract} mengen und Nutzungsart analysiert. Dazu wurden mit der Thermalformel nach Ingerle (1988), modifiziert durch Rauch (1992), entsprechend ÖWAVRegelblatt 207 die Temperaturausbreitungslängen und Strömungsbreiten jedes einzelnen Grundwassernutzers im Bearbeitungsgebiet berechnet. Zur Visualisierung des thermischen Einflusses auf das Grundwasser wurden diese in ARCMAP 10.1 grafisch dargestellt und mit im Feld durchgeführten Temperaturmessungen verglichen. Eine klare Überlagerung der Bereiche mit gemessener thermischer Beeinflussung des Grundwassers und jenen Bereichen mit rechnerisch sich überlagernden Wärmefahnen wurde dabei erkennbar. Basierend darauf wurden in einem nächsten Schritt unterschiedliche Varianten für mögliche Fernkältetrassen und zugehörige Kälteerzeugungsstandorte entwickelt. Dazu wurde zwischen zentralen (vollständige Aufschließung vorhandener Nutzer) und semi-zentralen Versorgungsvarianten (teilweise Aufschließung vorhandener Nutzer)

\section{S. Krakow $(\bowtie)$}

Assoc. Prof. DI Dr. techn. D. Fuchs-Hanusch Institut für Siedlungswasserwirtschaft und Landschaftswasserbau,

Technische Universität Graz, Stremayrgasse 10, $8010 \mathrm{Graz}$, Österreich

E-Mail: s.krakow@tugraz.at

\section{District cooling to avoid} groundwater warming and user conflicts using the example of the city Linz - Evaluation with ÖWAVnorm 207 and qualitative efficiency analysis

Abstract Groundwater investigations in Linz have shown, that the temperature in the groundwater reservoir has increased significantly compared to the temperature of the environment. To investigate whether the existing groundwater facilities for cooling have a quantifiable impact on groundwater temperatures all given permissions regarding groundwater usage for cooling premises were analyzed in detail. Therefore data on the yearly amount and type of groundwater usage were collected and sorted for further analyses. Based on these data the length and the width of the temperature flags in the groundwater reservoir were calculated with the thermal formula of Ingerle (1988), modified by Rauch (1992) and corresponding to the ÖVAW technical rule 207. The temperature flags of every groundwater user with cooling purposes were visualized with the software ARCMAP 10.1 and compared with real data from field measurements. Furthermore an analyses of uncertainty for the length of the temperature flags was involved in the study. With this method the areas where a thermic impact to the groundwater reservoir is of a high likelihood because of existing groundwater usage for cooling purposes were identified. Based on this in a next step different alternatives for district cooling were developed involving both central (covering all existing groundwater users) and semi central (covering only parts of existing users) solutions. To compare and evaluate the difference an efficiency analysis was undertaken. In this article the calculation and results of the thermal flag analysis of all relevant groundwater users are presented. Two of the seven alternatives for district cooling are then introduced more detailed. Further the efficiency analysis of the different alternatives is described using economical, ecological and social criteria.

\section{Einleitung}

Grundwasser ist eine wichtige Ressource für die Trinkwassererzeugung und in diesem Zusammenhang besonders schützenswert. Dies wird beispielsweise in $\S 30$ des Wasserrechtsgesetzes WRG (1959) definiert. So heißt es unter anderem „Insbesondere ist Grundwasser sowie Quellwasser so reinzuhalten, dass es als Trinkwasser verwendet werden kann. “( 30 WRG 1959). Neben der Trinkwasserversorgung besteht allerdings auch die Möglichkeit das Grundwasser thermisch zu nutzen, beispielsweise für die Kälteerzeugung.

Die Kälteerzeugung gewinnt an Bedeutung, da insbesondere im Zuge des Klimawandels ein steigender Kältebedarf anzunehmen ist (siehe Synthesis Report der IPCC 2014). Die Nutzung von Grundwasser zu diesem Zweck stellt eine umweltfreundliche und ressourcenschonende Möglichkeit dar, Gebäude und technische Anlagen zu kühlen. 


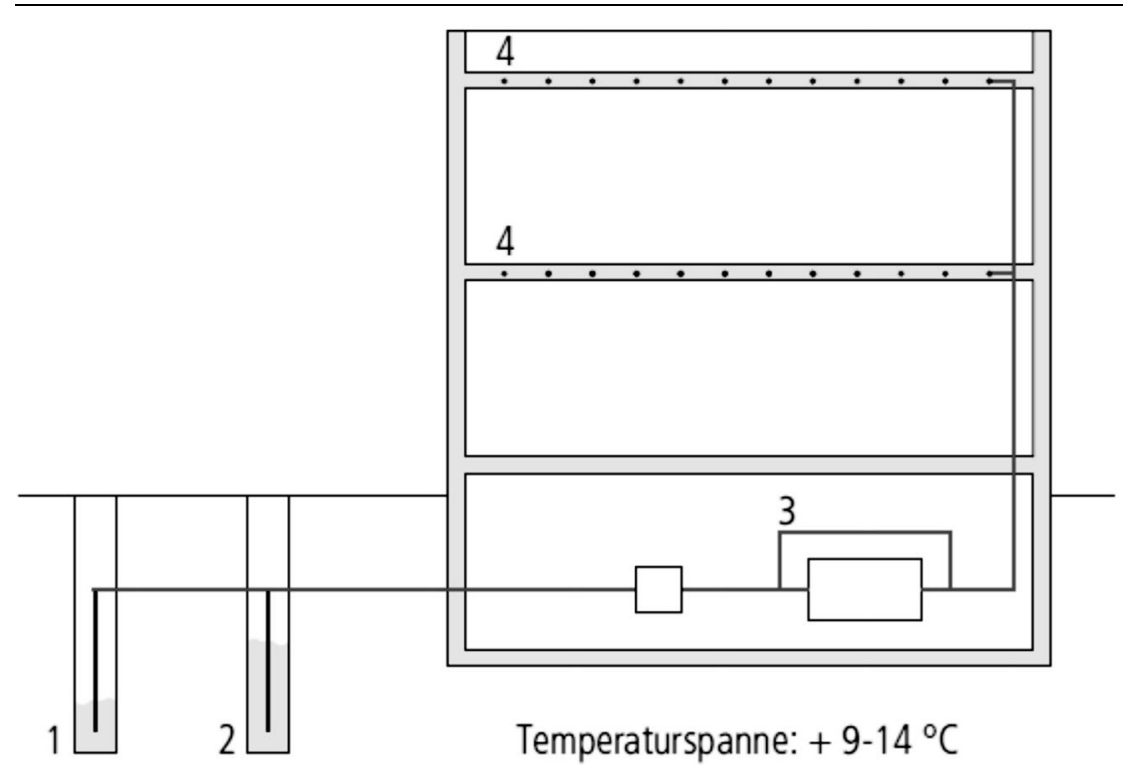

\section{Grundwassersaugbrunnen \\ 2 Grundwasserschluckbrunnen \\ 3 Reversible Wärmepumpe evtl. mit Bypass \\ 4 Flächenkühlsystem}

Abb. 1 Kälteerzeugung aus Grundwasser. (Quelle: Bohne 2014)

Bei der Kälteerzeugung aus Grundwasser werden die in der Regel niedrigen und konstanten Temperaturen des Grundwassers genutzt. Entsprechend ÖWAV-Regelblatt 207 (2009) liegt in Österreich die Temperatur in oberflächennahen, anthropogen unbeeinflussten Grundwässern in einer Tiefe von bis zu $7 \mathrm{~m}$ zwischen ca. 7 und $12{ }^{\circ} \mathrm{C}$. Die jahreszeitlich bedingten Temperaturschwankungen reichen bis in eine Tiefe von ca. 10 bis $20 \mathrm{~m}$. Die Grundwasserförderung erfolgt über einen Brunnen. Das vorhandene Temperaturpotenzial wird über Wärmetauscher-Anlagen abgegriffen. Das erwärmte Kühlwasser wird danach entweder über einen Schluckbrunnen ins Grundwasser versickert oder in ein Oberflächengewässer abgeleitet. Abb. 1 zeigt die schematische Darstellung einer Gebäudekühlung mit Grundwasser.

Thermisch genutztes Grundwasser sollte bei der Rückversickerung eine Temperatur von $5{ }^{\circ} \mathrm{C}$ nicht unterschreiten und eine Temperatur von $20{ }^{\circ} \mathrm{C}$ nicht überschreiten. Die maximale Aufwärmung bzw. Abkühlung des Grundwassers wird im ÖWAV-Regelblatt 207 (2009) mit 6 Kelvin definiert.
Wird das erwärmte Grundwasser nicht in den Grundwasserleiter zurückversickert, sondern in ein Fließgewässer abgeleitet, ist außerdem die Abwasseremissionsverordnung Kühlsysteme und Dampferzeuger - AEV (2003) zu beachten. In dieser ist beispielsweise geregelt, dass die Einleittemperatur in ein Fließgewässer $30{ }^{\circ} \mathrm{C}$ nicht überschreiten darf und gleichzeitig die maximale Aufwärmspanne $10 \mathrm{~K}$ beträgt.

Bei der Grundwasserentnahme zur Kälteerzeugung handelt es sich um die Benutzung eines Gewässers, die entsprechend § 10 WRG (1959) einer wasserrechtlichen Bewilligung bedarf. Details zum Bewilligungsverfahren sind in den $\$ \S 103-113$ WRG (1959) geregelt. So müssen z. B. der genaue Umfang und die Art der Auswirkungen auf das öffentliche Interesse geprüft und bei Bedarf entsprechende Schutzmaßnahmen festgelegt werden.

Mit der Bewilligung des Nutzungsrechtes, Grundwasser für Kühlzwecke entnehmen zu dürfen, gehen auch verschiedene Pflichten einher. So ist der Wasserberechtigte entsprechend $\S 50$ WRG (1959) zur Instandhaltung seiner Anlagen verpflichtet und muss diese in einem Zustand erhalten, sodass öffent- liche Interessen oder fremde Rechte nicht beeinflusst werden.

Weitere wesentliche Randbedingungen zur thermischen Nutzung von Grundwasser sind im bereits angesprochenen ÖWAV-Regelblatt 207 (2009) zu finden. Eine wichtige Festlegung ist, dass die derzeitige und zukünftige Versorgung der Bevölkerung mit Trinkwasser gegenüber der thermischen Nutzung uneingeschränkten Vorrang besitzt und das Grundwasser flächendeckend als Trinkwasser zu erhalten ist. Des Weiteren ist die thermische Nutzung von Grundwasser auf die oberflächennahen Grundwasservorkommen zu beschränken. Wird Grundwasser für Kühlwasserzwecke entnommen und nicht weiter als Nutzwasser verwendet, so ist es vollständig in den Grundwasserleiter zurückzuführen. Thermische Nutzungsanlagen dürfen in Schutzzone III von Schutzgebieten nur dann errichtet werden, wenn die Anlagen dem besonderen Schutzbedarf entsprechen, spezielle Begleitmaßnahmen verwendet werden und geeignete hydrogeologische Standortbedingungen gegeben sind. Außerdem besagt das ÖWAV-Regelblatt 207 (2009) auch, dass eine Verunreinigung des Grundwassers verlässlich verhindert werden muss. Demzufolge sind nur Wärmeträgermedien und Arbeitsmittel einzusetzen, die bei unbeabsichtigtem Austritt keine nachhaltig schädlichen Auswirkungen haben.

In der Stadt Linz werden bereits seit mehreren Jahren Anlagen zur Kälteerzeugung aus Grundwasser betrieben. Ein Teil dieser Anlagen versickert erwärmtes Kühlwasser in das Grundwasser zurück. Der andere Teil leitet erwärmtes Grundwasser in die Donau. In den Jahren 2004 und 2006 wurden von der DonauConsult Zottl \& Erber ZT GmbH Untersuchungen im Auftrag des Landes Oberösterreich zur Grundwasserbewirtschaftung Linz durchgeführt, dabei wurden im Vergleich $\mathrm{zu}$ den Umgebungstemperaturen erhöhte Grundwassertemperaturen festgestellt.

Das Institut für Siedlungswasserwirtschaft und Landschaftswasserbau der TU Graz wurde 2013 vom Amt der Oberösterreichischen Landesregierung beauftragt, die Grundwassernutzung zur Kälteerzeugung im Stadtgebiet Linz unter anderem zu quantifizieren sowie herauszuarbeiten, wie stark die bestehenden Kältenutzungsanlagen das Grundwasser im Stadtgebiet bereits beeinflussen. Mögliche aktuelle bzw. 


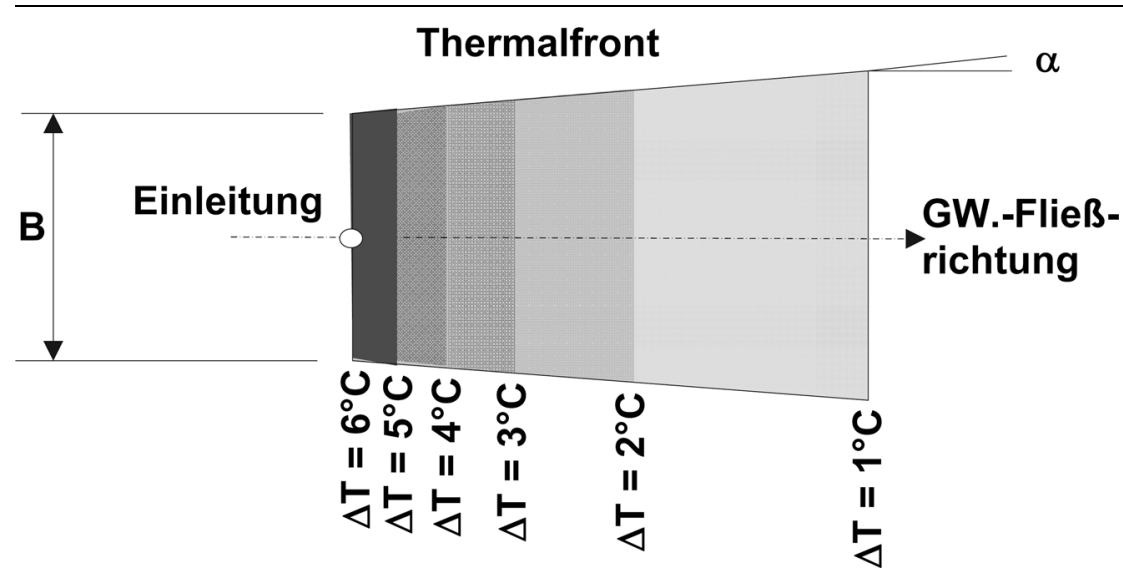

Abb. 2 Strömungsbild Temperaturfahne

zukünftige Nutzungskonflikte waren ebenfalls von Interesse. Hauptziel war es, Varianten zentraler Fernkältenutzungsanlagen als Alternative $\mathrm{zu}$ den zahlreichen vorhandenen Einzelanlagen $\mathrm{zu}$ beschreiben und basierend auf technischen, ökonomischen, sozialen und ökologischen Bewertungskriterien zu vergleichen (Strategische Studie, Fernkälte Linz - Modul I, Wasserwirtschaftliche Grundlagen 2013).

Unter einer zentralen Fernkälteversorgung ist ein zusammenhängendes Fernkälterohrnetz mit einer oder mehreren zentralen Kälteerzeugeranlagen, ähnlich einer Fernwärmeversorgung, zu verstehen. Für die Kälteerzeugung kann das bereits angesprochene Grundwasser genutzt werden oder klassische Kompressionskältemaschinen bzw. sogenannte Absorptionskälteanlagen, bei denen Wärme als Primärenergie genutzt wird.

\section{Methodik}

\subsection{Datenerhebung und Quantifizierung vorhandener Kältenutzer}

Um die aktuelle Grundwassernutzung hinsichtlich Kälteerzeugung quantifizieren zu können, wurden alle Kältenutzer auf Basis der wasserrechtlichen Bewilligungen im Bearbeitungsgebiet Linz ermittelt. Diesbezüglich wurden vom Amt der Oö. Landesregierung Unterlagen über vorhandene Bewilligungen, Art der Entnahme bzw. Rückführung sowie bewilligte Konsensmengen zur Verfügung gestellt. Auf Basis dieser Unterlagen sowie mithilfe des digitalen Oberösterreichischen Raum-Informations-Systems (DORIS) wurden alle aktuellen Wasserrechte, die für die gegenständlichen Untersuchun- gen von Relevanz waren, aufbereitet Dazu wurden u. a. Datensätze, die einem Grundwassernutzer zuzuordnen waren, aber aus unterschiedlichen Quellen stammten, in einer gemeinsamen Datei zusammengeführt, die Wasserrechte nach Entnahme, Versickerung und Ableitung aufgetrennt sowie die jeweiligen durchschnittlichen Jahreskonsensmengen aufsummiert. In diesem Zusammenhang wurde auch die geografische Lage der technischen Anlagen über Hoch- und Rechtswerte ermittelt. Zudem wurden die lokalisierten Kältenutzer angeschrieben und die im Kalenderjahr 2012 entnommenen bzw. versickerten Grundwassermengen abgefragt. Dadurch konnte ein erster Überblick über die tatsächliche Grundwassernutzung gewonnen werden.

\subsection{Berechnung der \\ Temperaturausbreitung im Grundwasser}

Zur Abschätzung und Visualisierung des Temperatureinflusses von versickertem, erwärmtem Grundwasser kam die Berechnung der Temperaturanomalien nach Ingerle (1988), modifiziert durch Rauch (1992) zur Anwendung, da für das Stadtgebiet Linz keine komplexeren Grundwassermodelle zur Verfügung standen.

Diese Methode ist ein vereinfachtes, analytisches Berechnungsverfahren und wird im ÖWAV-Regelblatt 207 (2009) beschrieben. Die Berechnung kann als Excel-Sheet auf der Homepage des ÖWAV (www.oewav. at/publikationen) unter dem Eintrag „ÖWAV-RB 207: Thermische Nutzung des Grundwassers und des Untergrunds" heruntergeladen werden.
Die Temperaturfahnen werden mit der Ausdehnungslänge L und der Strömungsbreite B trapezförmig dargestellt (siehe Abb. 2).

Das Ende der Temperaturanomalie ist dann erreicht, wenn der Temperaturunterschied zwischen umgebenden Grundwasser und Wärmefahne < 1 Kelvin beträgt. Die seitliche Ausbreitung wird mit dem Winkel $\alpha$ dargestellt und bildet Dispersionseffekte sowie die Verschwenkung der Grundwasserströmungsrichtung ab. Nach Rauch (1992) liegen die Werte für $\alpha$ zwischen 5 und $15^{\circ}$.

Für die Anwendung der Berechnungsmethode nach Ingerle sind verschiedene Randbedingungen zu beachten und folgende Annahmen zu treffen:

- Die Mächtigkeit H, die Durchlässigkeit $\mathrm{k}_{\mathrm{f}}$ und die Überdeckung A des Grundwasserfeldes sind näherungsweise konstant.

- Der Rückgabebrunnen ist als vollkommener Brunnen anzusehen (gleichmäßige Verteilung des Rückgabewassers über die gesamte Grundwassermächtigkeit).

- Der Entnahmebrunnen wird durch den Rückgabebrunnen nicht beeinflusst.

- Der Rückgabebrunnen wird kontinuierlich betrieben.

- Die Einleitung des Rückgabewassers in den Grundwasserkörper erfolgt im Bereich des Rückgabebrunnens ohne Vermischung mit der Strömungsbreite B.

Die detaillierten Beschreibungen $\mathrm{zu}$ den Randbedingungen der Ingerle-Methode sind ÖWAV-Regelblatt 207 (2009) zu entnehmen.

Die für die Berechnung der Temperaturfahnen notwendigen Formeln werden im Folgenden kurz beschrieben.

Die Strömungsbreite B wurde wie folgt ermittelt:

$$
B=\frac{Q}{k_{f} \cdot H \cdot J}
$$

Für die Infiltrationsmenge $\mathrm{Q}\left[\mathrm{m}^{3} / \mathrm{s}\right]$ wurde die durchschnittliche, jährliche Konsensmenge der in den Wasserrechten genehmigten Versickerungsmenge angesetzt. Vereinfachend wurde ein kontinuierlicher Betrieb über das ganze Jahr angesetzt. Der Durchlässigkeitsbeiwert $\mathrm{k}_{\mathrm{f}}[\mathrm{m} / \mathrm{s}]$ und die Grundwassermächtigkeit $\mathrm{H}[\mathrm{m}]$ wurden aus von der DonauConsult Zottl \& Erber ZT GmbH im Zuge der Studie (2004) zur Grundwasserbewirtschaftung Linz erstellten Plänen entnommen. Das Grundwas- 
Tab. 1 Übersicht Berechnungsparameter Temperaturfahnen nach Ingerle (1988), modifiziert durch Rauch (1992)

\begin{tabular}{|c|c|c|c|}
\hline Parameter & Einheit & Beschreibung & Quelle für die Berechnung \\
\hline $\mathrm{T}_{\mathrm{i}}$ & {$\left[{ }^{\circ} \mathrm{C}\right]$} & $\begin{array}{l}\text { Temperatur an Stelle i im Abstand L von der } \\
\text { Einleitstelle }\end{array}$ & Teil der Iterationsberechnung \\
\hline $\mathrm{T}_{0}$ & {$\left[{ }^{\circ} \mathrm{C}\right]$} & durchschnittliche Entnahmetemperatur & $\begin{array}{l}\text { Studie zur Grundwasserbewirtschaftung, DonauConsult Zottl \& Erber } \\
\text { ZT GmbH (2006) }\end{array}$ \\
\hline $\mathrm{T}_{\mathrm{A}}$ & {$\left[{ }^{\circ} \mathrm{C}\right]$} & Rückgabetemperatur & $\begin{array}{l}\text { aus Wasserbuch-Evidenz oder Festlegung auf } 18{ }^{\circ} \mathrm{C} \text { in Abstimmung } \\
\text { mit Amt der 0ö. Landesregierung }\end{array}$ \\
\hline$\alpha$ & {$[-]$} & seitlicher Ausbreitungswinkel der Thermalfront & entsprechend ÖWAV RB 207 (2009) auf $6^{\circ}$ festgelegt \\
\hline$B_{i}$ & [m] & Breite der Temperaturanomalie an der Stelle i & Teil der Iterationsberechnung \\
\hline wi & {$[-]$} & Hilfsgröße an derStelle i & Teil der Iterationsberechnung \\
\hline$\Delta x$ & [m] & Länge des iterativen Schrittes in $x$-Richtung & auf $5 \mathrm{~m}$ festgelegt \\
\hline A & [m] & Flurabstand & $\begin{array}{l}\text { Studie zur Grundwasserbewirtschaftung, DonauConsult Zottl \& Erber } \\
\text { ZT GmbH (2004) }\end{array}$ \\
\hline Cvw & {$\left[\mathrm{J} / \mathrm{m}^{3} / \mathrm{K}\right]$} & spezifische Wärmekapazität von Wasser & $C_{V w}=4,2^{*} 10^{6} \mathrm{~J} / \mathrm{m}^{3} / \mathrm{K}$ \\
\hline$\lambda_{d}$ & {$[\mathrm{~W} / \mathrm{m} / \mathrm{K}]$} & Wärmeleitfähigkeit der Deckschicht & entsprechend ÖWAV RB 207 (2009) $\lambda_{D}=0,5 \mathrm{~W} / \mathrm{m} / \mathrm{K}$ gewählt \\
\hline
\end{tabular}

serspiegelgefälle $\mathrm{J}^{[-]}$basiert auf der Grundlage des Grundwasserschichtenplanes der Stadt Linz (Prexel, Mittelwasser).

Die Ausdehnungslänge L der Temperaturfahne ist iterativ zu berechnen. Wichtig dabei ist die Wahl der Länge des iterativen Berechnungsschrittes $\Delta \mathrm{x}$. Dieser sollte so gewählt werden, dass sich durch eine Variation von $\Delta \mathrm{x}$ die Thermalfahne nicht mehr wesentlich ändert. Danach erfolgt die schrittweise Berechnung der Temperatur an der Stelle i entsprechend Gl. 2. Mit Gl. 3 wird die Strömungsbreite an der Stelle i ermittelt und mit Gl. 4 die Hilfsgröße w an der Stelle i (jeweils in Abhängigkeit von $\Delta \mathrm{x}$ ). Abbruchkriterium für die Iteration ist ein Temperaturunterschied von $<1 \mathrm{~K}$ zwischen der Temperaturfahne und dem umgebenden Grundwasser. Die Ausdehnungslänge L entspricht dann der Anzahl der Iterationsschritte multipliziert mit dem Berechnungsschritt $\Delta \mathrm{x}$.

$$
\begin{aligned}
& T_{i+1}= \\
& \frac{T_{i}\left(B_{i}-\frac{w_{i}}{2}\right)+T_{0} \cdot\left(2 \cdot \Delta x \cdot \tan \alpha+w_{i}\right)}{\left(B_{i+1}+\frac{w_{i}}{2}\right)} \\
& B_{i+1}=B_{i}+2 \cdot \Delta x \cdot \tan \alpha
\end{aligned}
$$

$$
\frac{\lambda_{D}}{\left(A+\frac{H}{4}\right)} \cdot\left(B_{i}+\Delta x \cdot \tan \alpha\right) \cdot \Delta x \cdot \frac{1}{\left(\frac{Q}{B}\right) \cdot c_{v w}}
$$

Die verwendeten Berechnungsparameter sind in Tab. 1 zusammengefasst und beschrieben.

Nach Berechnung der Temperaturfahnen wurden diese mit Hilfe von ARCMAP 10.1 in Anlehnung an Abb. 2 visualisiert. Dazu wurden Skripts basierend auf Python 2.7 erstellt. Ge- genseitige Beeinflussungen zwischen den Grundwassernutzen wurden bei den Berechnungen nicht betrachtet. Die Fließrichtung wurde anhand der Grundwasserstandshöhenlinien ermittelt. Bei Kältenutzern, die mehrere Brunnen für die Versickerung betreiben, wurde die Gesamtversickerungsmenge (Konsensmenge) auf die Anzahl der Versickerungsbrunnen aufgeteilt, aber nur eine Wärmefahne dargestellt. Dies ermöglichte einen Überblick über Ausbreitung und Fließrichtung der Wärmefahnen im Bearbeitungsgebiet. Auf dieser Grundlage wurde in einem zweiten Berechnungsschritt die Fließrichtung der Temperaturanomalien an den Verlauf der Grundwasserstandshöhenlinien angepasst. In einem dritten Schritt wurden der $\mathrm{k}_{\mathrm{f}}$-Wert und das Grundwasserspiegelgefälle $\mathrm{J}$ über den gesamten Fließweg gemittelt und die Temperaturfahnen mit diesen Werten erneut berechnet.

Des Weiteren wurde eine Unsicherheitsanalyse hinsichtlich der Ausdehnungslängen der Temperaturfahnen durchgeführt. Dies erfolgte durch Variation der Parameter Grundwassermächtigkeit $\mathrm{H}$ und Ausbreitungswinkel $\alpha$. Nach Sitzenfrei und Rauch (2014) ist die Grundwassermächtigkeit $\mathrm{H}$ als sensitivster Eingangswert, bezogen auf die Ausbreitungslänge L, zu betrachten. Der Ausbreitungswinkel $\alpha$ und die Wärmeleitfähigkeit der Deckschicht $\lambda_{D}$ werden im ÖWAV-Arbeitsbehelf 43 (2014), dem im Jahr 2014 veröffentlichten Leitfaden zur Anwendung der Thermalfahnenformel nach ÖWAV-Regelblatt 207 (2009), als weitere wichtige Eingangsparameter beschrieben. Die Wärmeleitfähigkeit der Deckschicht wurde allerdings bei der Unsicherheitsanalyse nicht weiter betrachtet, da keine gesicherten Erkenntnisse über mögliche Bandbreiten von $\lambda_{\mathrm{D}}$ vorlagen.

Von Sitzenfrei und Rauch (2014) wurde zudem die sogenannte thermische Tiefe eingeführt, die die für die Ausbreitung der Wärmefahnen maßgebende Grundwassermächtigkeit durch eine empirische Formel näher beschreibt. Detaillierte Ausführungen diesbezüglich liefert der ÖWAV-Arbeitsbehelf 43 (2014). Für die im Bearbeitungsgebiet lokalisierten Kältenutzer wurde jeweils die thermische Tiefe berechnet. Der geringste Wert für die thermische Tiefe (über alle Nutzer) lag bei 2,2 m. Um dies bei der Unsicherheitsanalyse zu berücksichtigen, wurde die untere Grenze für die Variation der Grundwassermächtigkeit $\mathrm{H}$ auf $2 \mathrm{~m}$ festgelegt. Beim Ausbreitungswinkel $\alpha$ erfolgte eine Variation zwischen 5 und $15^{\circ}$. Beide Parameter wurden jeweils 10.000 Mal zufällig und gleichverteilt generiert. Für jeden Kältenutzer wurden so 10.000 WertePaare ermittelt und mit diesen jeweils die Ingerle-Berechnung wiederholt. Die Ergebnisunsicherheit aufgrund der gewählten Eingangsparameterunsicherheit wurde mittels Berechnung von Mittelwert und Standardabweichung bewertet.

\subsection{Entwicklung von}

\section{Fernkälteversorgungsvarianten}

Im Hinblick auf eine zentrale Versorgung mit Kälte wurden 7 Fernkälteversorgungsvarianten mit unterschiedlichen Netzverläufen sowie unterschiedlichen Standorten für die Kältegewinnung entwickelt. Es wurden 4 zentrale und 3 semi-zentrale Varianten unterschieden. Bei den zen- 


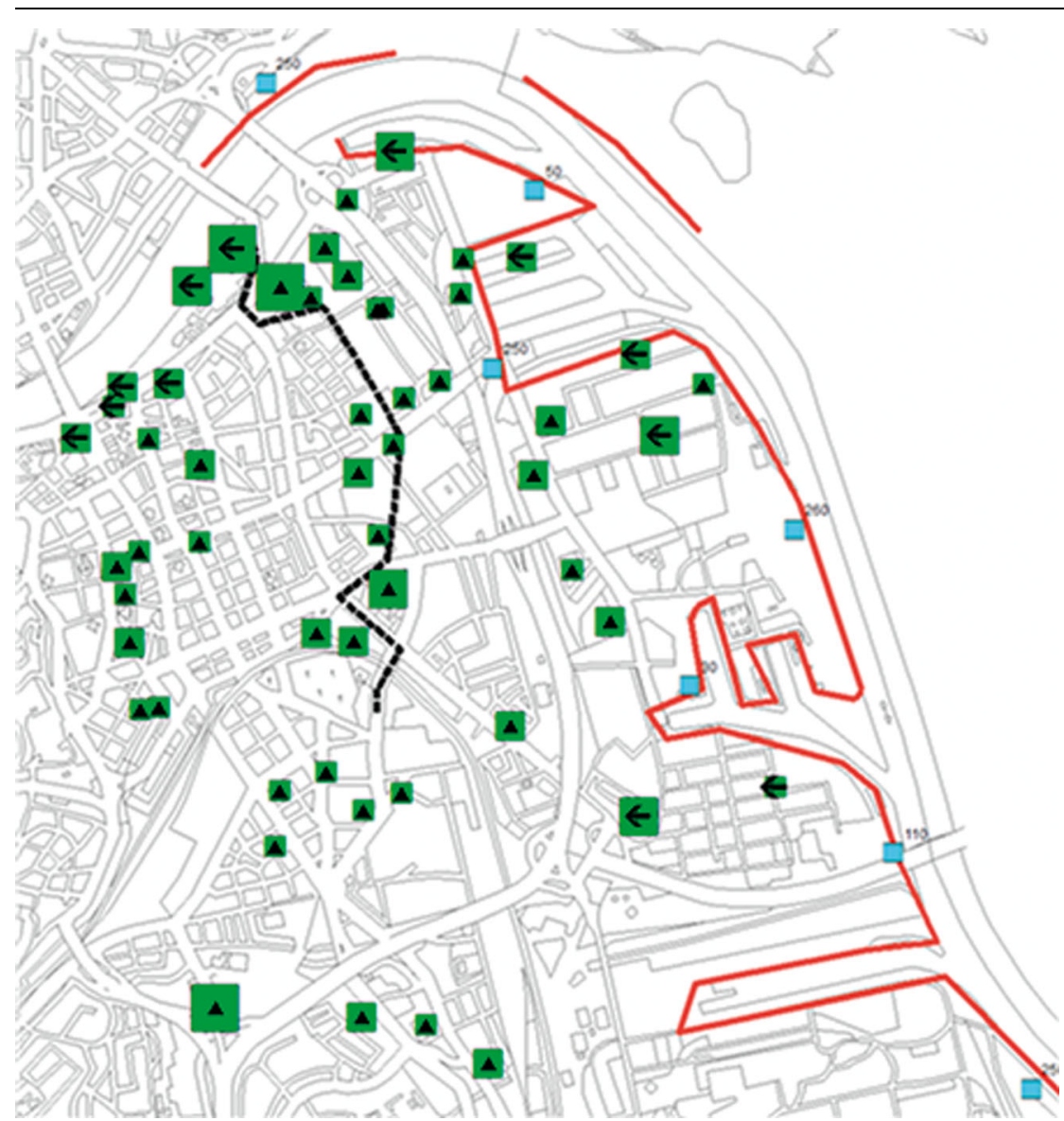

Abb. 3 Betrachtungsgebiet und Kältenutzer

tralen Varianten sollten alle bekannten Kältenutzer mit Fernkälte erschlossen werden, wohingegen bei den semi-zentralen Varianten Einzelnutzer weiterhin möglich waren. Eine Variante konnte aus mehreren voneinander unabhängigen Netztrassen bestehen. Die Festlegung der Leitungstrassen erfolgte GISbasierend und ohne Überprüfung der bereits im Straßenbereich vorhandenen Leitungstrassen.

Für zentrale Kälteerzeugungsanlagen wurden verschiedene Möglichkeiten in Betracht gezogen. Zum einen eine zentrale Kälteerzeugung aus Grundwasser mit Rückversickerung des erwärmten Kühlwassers sowie die Nutzung von vorhandenen Pumpenanlagen, die bereits kontinuierlich Grundwasser fördern und in die Donau ableiten. Betreiber solcher Anlagen war z. B. die Austrian Hydro Power AG (AHP). Hintergrund war die Abdichtung des Grundwasserleiters zur Donau im Bearbeitungsgebiet der Stadt Linz und der aus diesem Grund notwendigen Grundwasserhaltungsmaßnahmen. Die Abdichtung zur Donau erfolgte im Zuge
Kompressionskältemaschinen vorzusehen.

\subsection{Nutzwertanalyse zur Bewertung der} Fernkälteversorgungsvarianten

Um die verschiedenen Varianten miteinander $\mathrm{zu}$ vergleichen, wurde eine Nutzwertanalyse durchgeführt. Dabei handelt es sich um eine qualitative, nicht monetäre Analysemethode der Entscheidungstheorie. Für die Bewertungsmatrix wurden 4 Hauptkriterien, zur ökologischen Bewertung (Wasserwirtschaft/ Umwelt), zur ökonomischen Bewertung (Sicherung Wirtschaftsstandort), zur rechtlich/ sozialen Bewertung (Wasserrechte/ Fremde Rechte) sowie zur technischen Bewertung (Umsetzbarkeit) mit jeweils 2 bis 4 Unterpunkten entwickelt (Tab. 2). Als ein technischer Bewertungsparameter wurde beispielsweise die Kennzahl „Trasseneffizienz“, gemessen in $\left[\mathrm{m}^{3} / \mathrm{m}\right]$, eingeführt. Diese Kennzahl ist als Maß für die Versorgungsdichte einer Variante anzusehen. Dabei wurde die mittlere, aufsummierte und genehmigte Jahreskonsensmenge der angeschlossenen Kältenutzer auf die entsprechende Netzlänge bezogen.

Jede Variante wird entsprechend einer zuvor festgelegten Skala $(1-$ sehr negativ, 2 - negativ, 3 - neutral, 4 - positiv, 5 - sehr positiv) bewertet. Der Bewertungsfaktor und der Gewichtungsfaktor werden multipliziert. Alle Einzelergebnisse werden danach aufsummiert. Die Variante mit der höchsten Bewertungszahl ist als Vorzugsvariante anzusehen.

\section{Ergebnisse} kältezentralen wurde rein bilanziell hinsichtlich der genehmigten Jahresdurchschnittsmengen der Kältenutzer durchgeführt. Es erfolgte keine Betrachtung von Wärmefrachten bzw. Kälteleistungen, da der tatsächliche Leistungsbedarf der einzelnen Kälteanlagen im Zuge dieses Projektes nicht ermittelt werden konnte. Ein Ansetzen der theoretisch möglichen maximalen Temperaturspreizung für jeden Kältenutzer würde zu deutlich erhöhten $\mathrm{Ge}$ samtleistungen führen und stellte keine Alternative dar. Die angesetzten Fernkältezentralen sollten demzufolge den durchschnittlichen Bedarf abdecken. Außerdem ist bei zentraler Kälteversorgung davon auszuggehen, dass sich die Lastabnahme vergleichmäßigt. Für den Spitzenbedarf sind ggf. zusätzliche

\subsection{Lokalisierung und Quantifizierung von vorhandenen Kältenutzern}

In Abb. 3 ist das Bearbeitungsgebiet mit den lokalisierten Kältenutzern in der Stadt Linz dargestellt.

Die grünen Quadrate entsprechen den genehmigten Grundwasserentnahmen. Dreiecke in den grünen Quadraten symbolisieren Grundwasserversickerungen, Pfeile Grundwasserableitungen in die Donau. Die blauen Quadrate sind Pumpstationen der Austrian Hydro Power AG, die kontinuierlich Grundwasser in die Donau ableiten. Die rote Begrenzungslinie im Donauuferbereich ist das Dichtungs- und Drainagesystem zwischen Grundwasserleiter und Donau. Des Weiteren war 


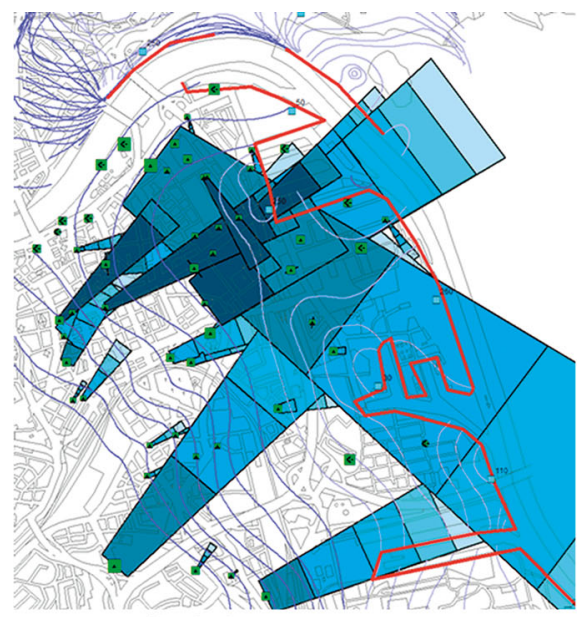

erster Schritt

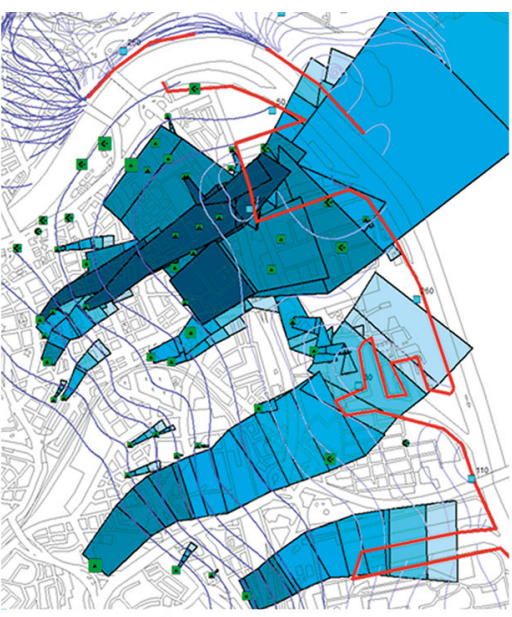

zweiter Schritt

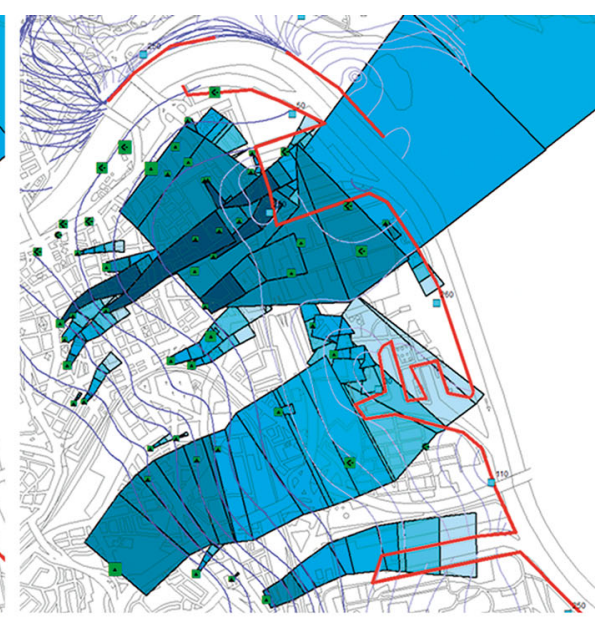

dritter Schritt

Abb. 4 Berechnete Temperaturfahnen-schrittweise Anpassung

Tab. 2 Bewertungskriterien und Gewichtung aus Nutzwertanalyse

\begin{tabular}{|c|c|c|c|}
\hline \multicolumn{2}{|l|}{ Wasserwirtschaft/ Umwelt } & \multicolumn{2}{|c|}{$\begin{array}{l}\text { Beitrag zur Sicherung des Wirtschafts- } \\
\text { standortes }\end{array}$} \\
\hline $\begin{array}{l}\text { Einfluss auf den Grundwasser- } \\
\text { stand }\end{array}$ & $10 \%$ & $\begin{array}{l}\text { sichere Versorgung vorhande- } \\
\text { ner Kältenutzer }\end{array}$ & $15 \%$ \\
\hline $\begin{array}{l}\text { Einfluss auf die Grundwasser- } \\
\text { temperatur }\end{array}$ & $10 \%$ & $\begin{array}{l}\text { Stabilisierung und Verbesse- } \\
\text { rung der Kühlleistung }\end{array}$ & $10 \%$ \\
\hline $\begin{array}{l}\text { Verringerung der } \\
\mathrm{CO}_{2} \text {-Emissionen }\end{array}$ & $5 \%$ & $\begin{array}{l}\text { Erweiterung von Kältenutzun- } \\
\text { gen/ zusätzliche Kältenutzer }\end{array}$ & $10 \%$ \\
\hline Energiebedarf & $5 \%$ & - & - \\
\hline \multicolumn{2}{|c|}{ Wasserrechte/ Fremde Rechte } & \multicolumn{2}{|l|}{ Umsetzbarkeit } \\
\hline $\begin{array}{l}\text { Konfliktpotential mit vorhande- } \\
\text { nen }\end{array}$ & $10 \%$ & $\begin{array}{l}\text { Synergie mit geplanter Straßen- } \\
\text { bahntrasse }\end{array}$ & $10 \%$ \\
\hline $\begin{array}{l}\text { Einfluss auf andere Grundwas- } \\
\text { sernutzungen }\end{array}$ & $5 \%$ & Trasseneffizienz & $10 \%$ \\
\hline
\end{tabular}

zum Zeitpunkt der Durchführung dieser Untersuchungen der Neubau einer Straßenbahnstrecke geplant, von der ein Teil der Trasse unterirdisch gebaut werden sollte (schwarz-gestrichelte Linie).

Insgesamt wurden im Bearbeitungsgebiet 54 Kältenutzer lokalisiert, die Grundwasser für Kühlzwecke entnehmen und danach erwärmt versickern bzw. in die Donau ableiten. Die durchschnittliche Jahreskonsensmenge lag bei ca. 585 l/s und der genehmigte Spitzenwert bei ca. 1.230 l/s. Die genehmigten Versickerungsmengen lagen durchschnittlich bei ca. 315 l/s und in der Spitze bei ca. 680 l/s (ungefähr zwei Drittel der Entnahmemenge). Im Durchschnitt durften ca. 284 l/s und in der Spitze ca. 405 l/s (ca. ein Drittel) in die Donau abgeleitet werden.

\subsection{Berechnung und Visualisierung der Temperaturfahnen - Basis: bewilligter Konsens}

Für die in Abb. 3 dargestellten Kältenutzer, die das erwärmte Grundwasser in den Grundwasserleiter zurückversickern, wurden die Temperaturfahnen ermittelt, wie in Abschn. 2.2 beschrieben. In Abb. 4 sind diese dargestellt. Aus konstruktiven Gründen laufen einige der Temperaturfahnen über die vorhandenen Dichtwände zur Donau hinweg. Tatsächlich ist dies nicht der Fall und der verwendeten Methodik geschuldet. Die farblichen Abstufungen stehen jeweils für $1 \mathrm{~K}$ Abkühlung über den Fließweg. Berechnungsschritt 1 und Berechnungsschritt 2 unterscheiden sich deutlich in Bezug auf die Fließrichtungen der Temperaturfahnen, die an die Grundwasserstandshöhenlinien angepasst wurden. Beim dritten Berechnungsschritt sind im Vergleich zum ersten und zweiten Berechnungsschritt deutliche Änderungen bei Länge und Breite der Temperaturfahnen zu erkennen.

Bei allen 3 Übersichtsplänen sind dieselben Tendenzen zu erkennen. Im Stadtgebiet nördlich des Hauptbahnhofes und in Höhe des Handelshafens sind Temperaturfahnen mit starken Überschneidungen und hohen Versickerungstemperaturen $\mathrm{zu}$ beobachten. Hier sind gegenseitige thermische Beeinflussungen sehr wahrscheinlich. Außerdem ist $\mathrm{zu}$ erkennen, dass die Pumpstationen der AHP erheblichen Einfluss auf die Fließrichtung der Temperaturfahnen haben.

In Abb. 5 sind die interpolierten Maximalwerte der Grundwassertemperaturmessungen aus den Jahren 2003 bis 2005 (Studie DonauConsult Zottl \& Erber $\mathrm{GmbH}$ 2006) dargestellt. Ausgehend von der Temperaturverteilung, sind ähnliche Temperaturbeeinflussungen wie in Abb. 4 erkennbar. Insbesondere fallen die bereits erwähnten Stadtbereiche durch stark erhöhte Grundwassertemperaturen $\left(>16{ }^{\circ} \mathrm{C}\right)$ auf.

Abb. 6 zeigt die aus der Unsicherheitsanalyse hervorgegangenen Temperaturfahnen für den Mittelwert und die positive bzw. negative Standardabweichung. Die Temperaturfahnen für den Mittelwert sind mit dem dunkelsten Blau (Himmelblau), die Temperaturfahnen für den Mittelwert plus Standardabweichung (die größten Fahnen) mit dem hellsten Blau (Sodalithblau) und die Temperaturfahnen für den Mittelwert minus Standardabweichung (die kleinsten Fahnen) mit Apatitblau dargestellt. Der Einfluss des Ausbreitungswinkels $\alpha$ und der Grund- 


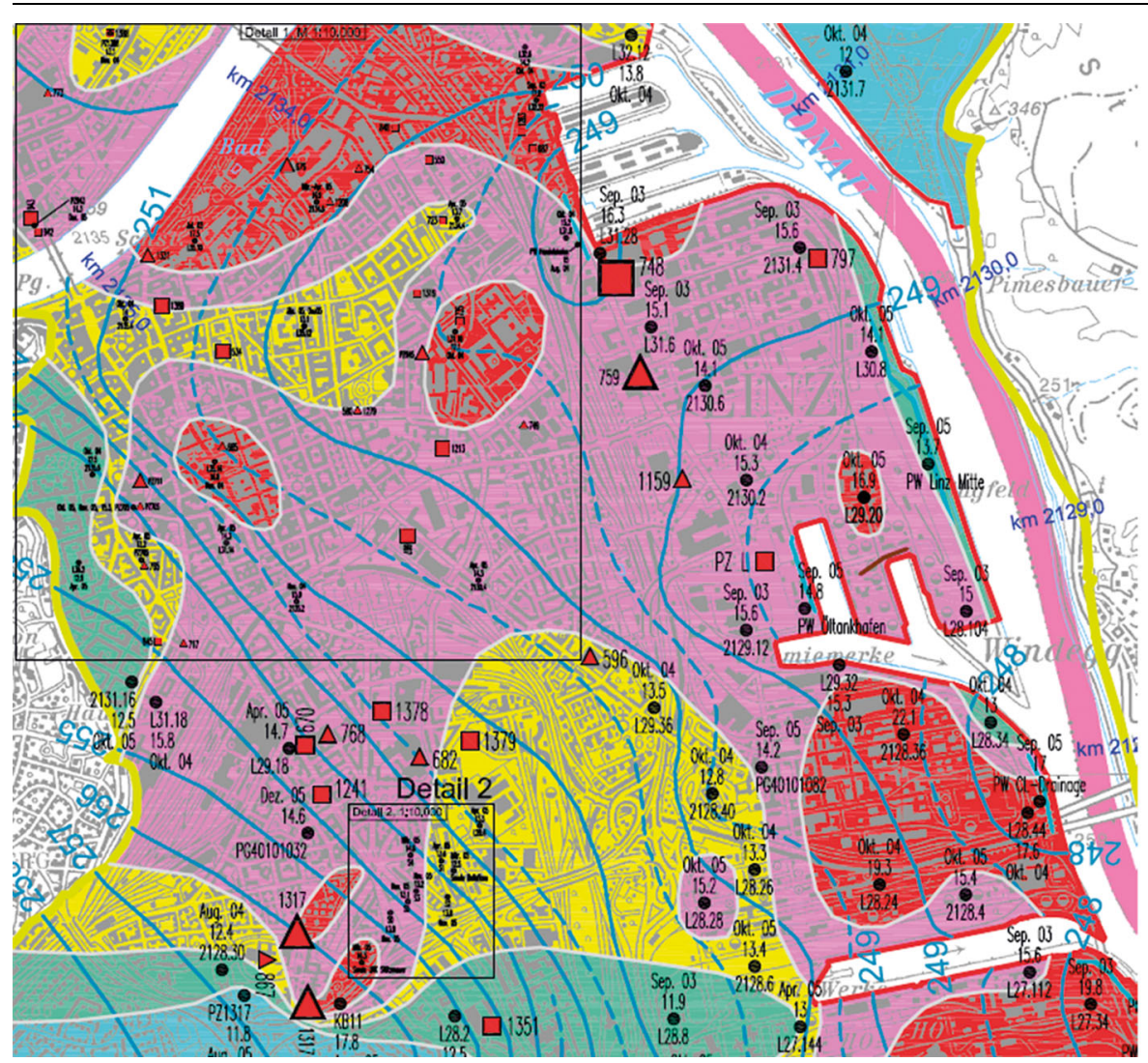

\section{LEGENDE:}

\section{Grundwassertemperatur}

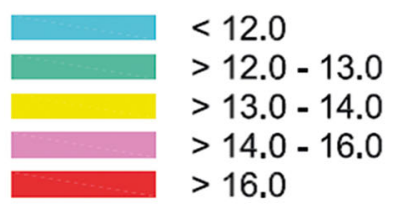

Abb. 5 Temperaturmessungen 2003 bis 2005, Maximalwerte. (Quelle: DonauConsult Zottl \& Erber GmbH 2006)

wassermächtigkeit $\mathrm{H}$ auf die Ausdehnungslänge $\mathrm{L}$ der Temperaturfahnen ist deutlich erkennbar, insbesondere bei den größeren Anlagen.

In Abb. 7 sind die Längen der Temperaturfahnen gegen die jeweiligen Versickerungsmengen aufgetragen. Dabei wird erkennbar, dass die Längen der Temperaturfahnen aus Berechnungsschritt 3 bei den kleineren Anlagen zum Großteil im Bereich des Mittelwertes und der zugehörigen Standardabweichung aus der Unsicherheitsanalyse liegen. Bei den größeren Anlagen (Versickerungsmenge $>5,6 \mathrm{l} / \mathrm{s}$ ) liegen die Temperaturfahnen nicht mehr in der Bandbreite der Standardabweichung. Eine mögliche Ursache dafür kann die Festlegung des Ausbreitungswinkels $\alpha$ auf $6^{\circ}$ im Berechnungsschritt 3 sein. Bei der Unsicherheitsanalyse erfolgte für $\alpha$ eine zufällige und gleichverteilte Variation zwischen 5 und $15^{\circ}$. Mit $\alpha=6^{\circ}$ liegt der Ausbreitungswinkel im unteren Bereich dieser Schwankungsbreite und führt gegenüber dem Mittelwert aus der Unsicherheitsanalyse zu höheren Ausbreitungslängen (geringere Verschwenkung des Grundwassers, geringere Dispersion). Außerdem war er- kennbar, dass die Ergebnisunsicherheit sowohl der Temperaturfahnenbreite als auch der Temperaturfahnenlänge, alleine aufgrund der Parametervariation von $\alpha$ und $H$, von Nutzer zu Nutzer sehr stark schwankt. Die Standardabweichung der Temperaturfahnenlängen lag zwischen $11 \%$ und $47 \%$. Eindeutige Korrelationen zwischen dieser stark streuenden Unsicherheit und einzelnen Eingangsparametern konnten nicht festgestellt werden was auf die große Interaktion der Eingangsparameter in Hinblick auf die Ergebnisvariabilität zurückgeführt werden kann.

Die Ermittlung von Temperaturfahnen nach Ingerle, modifiziert durch Rauch (ÖWAV-Regelblatt 207 2009) ist grundsätzlich ein einfacher Ansatz, um einen ersten Überblick über den Einfluss einzelner Kältenutzer auf die Grundwassertemperatur zu schaffen. Die Datengrundlage hat allerdings einen wichtigen Einfluss auf die Genauigkeit der Ergebnisse. Die Länge und Ausdehnung der berechneten Temperaturfahnen sollten eher als Anhaltspunkt gewertet werden, da die mit der Methode nach Ingerle nicht erfassten Einflüsse entlang des Fließweges einen weiteren maßgeblichen Einfluss auf das Endergebnis haben. Sind komplexe Sachverhalte zu betrachten und besteht die Möglichkeit, detaillierte Grundwassermodelle zu nutzen, sollten diese bevorzugt werden, auch im Hinblick auf die Vereinfachungen und Annahmen bei der Ingerle-Methode hinsichtlich gegenseitiger Beeinflussung von Nutzern.

\subsection{Berechnung und Visualisierung der Temperaturfahnen - Basis:}

Angaben aus Nutzerbefragung

Für die abgefragten Grundwasserentnahmemengen aus dem Kalenderjahr 2012 wurden ebenfalls die Temperaturfahnen nach Ingerle berechnet und grafisch aufbereitet (siehe Abb. 8).

Von den 54 angeschriebenen Kältenutzern haben 38 Nutzer Daten zur Verfügung gestellt. Bezogen auf die genehmigte Jahreskonsensmenge aller 54 Nutzer entsprach dies $64 \%$. Im Vergleich zu den vorherigen Berechnungen sind deutlich geringere Temperaturfahnen zu erkennen. Trotzdem lassen sich auch hier Überschneidungen bei den Temperaturfahnen nördlich des Bahn- 


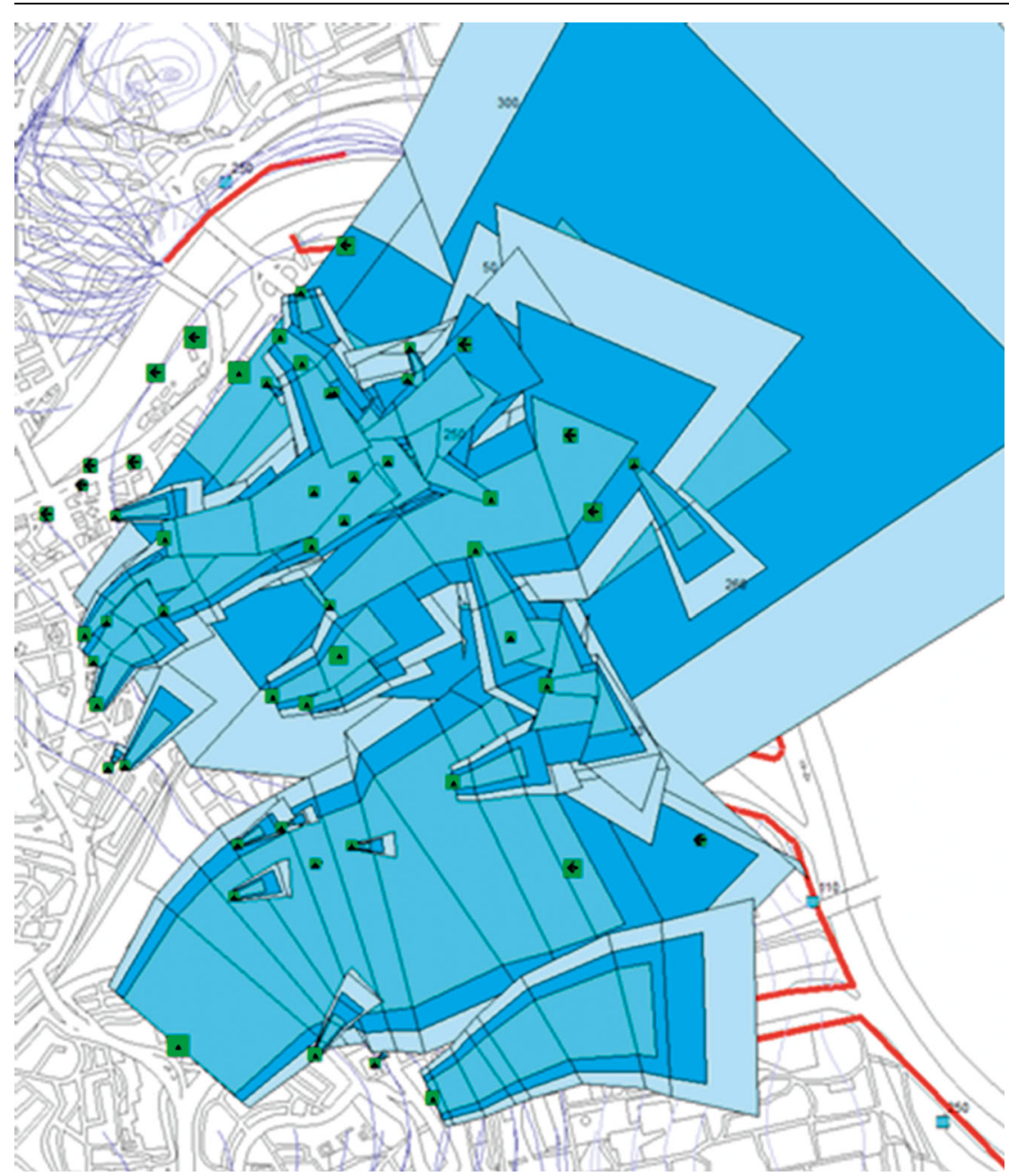

Abb. 6 Unsicherheitsanalyse-Schwankungsbreiten Temperaturfahnen

hofs und in Höhe des Handelshafens erkennen. Hier sind demzufolge thermische Beeinflussungen zu erwarten.

Von den Kältenutzern wurden meistens Jahresmengen zurückgemeldet, sodass Abb. 8 keine Maximalfälle darstellt. Außerdem wurden einige der Anlagen erst 2013 wieder in Betrieb genommen (4 Anlagen) und es ist anzunehmen, dass der thermische Einfluss durch die Grundwassernutzung für Kühlzwecke höher ist.

\subsection{Varianten zur Fernkälteversorgung - Eine Auswahl}

Im Folgenden werden 2 der 7 entwickelten Fernkältevarianten vorgestellt, die die Verringerung des Temperatureinflusses durch die Kältenutzung zum Ziel hatten.

Abb. 9 zeigt beispielhaft Variante 1 . Diese besteht aus 3 Netztrassen (Trasse A, Trasse B und Trasse C im blauen, roten bzw. gelben Bereich). Die Kälteerzeugung erfolgt über 4 Zentralen (jeweils als ausgefüllter Kreis dargestellt). Die nördliche Erzeugeranlage ist die vorhandene Fernkältezentrale der Linz AG, die westlichste Fernkältezentrale nutzt Grundwasser zur Kälteerzeugung. Das erwärmte Grundwasser wird wiederum versickert. Im Bereich der gelben Trasse befindet sich ein Pumpwerk der AHP (Ableitung von Grundwasser in die Donau). In diesem Bereich ist eine Fernkältezentrale vorgesehen, die bereits gepumptes Wasser für die Kälteerzeugung nutzen soll. Die anderen beiden Zentralen nutzen Grundwasser und versickern es jeweils erwärmt wieder. Die Gesamttrassenlänge beträgt $17 \mathrm{~km}$, die Trasseneffizienz $972 \mathrm{~m}^{3} / \mathrm{m}$. Die geplante Strassenbahntrasse kann zum Großteil für die Trassierung der Fernkälteleitungen genutzt werden, sodass hier Synergien hinsichtlich der Baukosten möglich sein sollten.
Abb. 10 zeigt die nach der bereits vorgestellten Methodik (Ingerle, modifiziert durch Rauch) berechneten Temperaturanomalien für die Fernkältezentralen, die Grundwasser erwärmt versickern. Angesetzt wurde die Summe der jährlichen Konsensmengen der angeschlossenen Kältenutzer. Als Versickerungstemperatur wurde jeweils $18{ }^{\circ} \mathrm{C}$ verwendet. Deutlich erkennbar ist die massive und weitläufige Ausbreitung der beiden Temperaturfahnen, was auf die deutlich erhöhten Versickerungsmengen zurückzuführen ist. Bei dieser Variante würde es zu einer weiträumigen thermischen Beeinflussung des Grundwasserleiters kommen. Zudem würde die Erzeugeranlage der Trasse C (roter Bereich) ebenfalls thermisch beeinflusst werden und damit weniger effektiv hinsichtlich des nutzbaren Temperaturpotenzials sein. Diese Variante war bei der Nutzwertanalyse eine der weniger erfolgreichen Varianten.

Abb. 11 zeigt Variante 6, die aus der Nutzwertanalyse hervorgegangene Vorzugsvariante.

Bei dieser Variante handelt es sich um eine semi-zentrale Variante mit einem zentralen Netz. Nicht alle Kältenutzer werden an das zentrale Netz angeschlossen (südlicher Bereich des Bearbeitungsgebietes). Für die Kälteerzeugung werden 3 Fernkältezentralen benötigt. Zum einen die vorhandene Fernkältezentrale der Linz AG, des Weiteren eine Anlage in der Nähe der vorhandenen Pumpstation der AHP und bei der dritten Anlage kann Grundwasser verwendet werden, welches erwärmt zurückinfiltriert wird, oder eine klassische Kälteerzeugung über Kompressionskälteanlagen erfolgen. Die Netzlänge bei dieser Variante beträgt ca. 13,1 km und die Trasseneffizienz liegt bei $1.114 \mathrm{~m}^{3} / \mathrm{m}$. Die geplante Straßenbahntrasse kann zum Großteil genutzt werden. Abb. 12 zeigt die Temperaturfahnen der nicht an das Fernkältesystem angeschlossenen Einzelnutzer. Die Zentralanlagen nutzen bereits bestehende Pumpwerke und leiten in die Donau ab. Die Temperaturbeeinflussung des Grundwassers ist bei dieser Variante deutlich geringer, als im Originalzustand. 


\section{Originalarbeit}
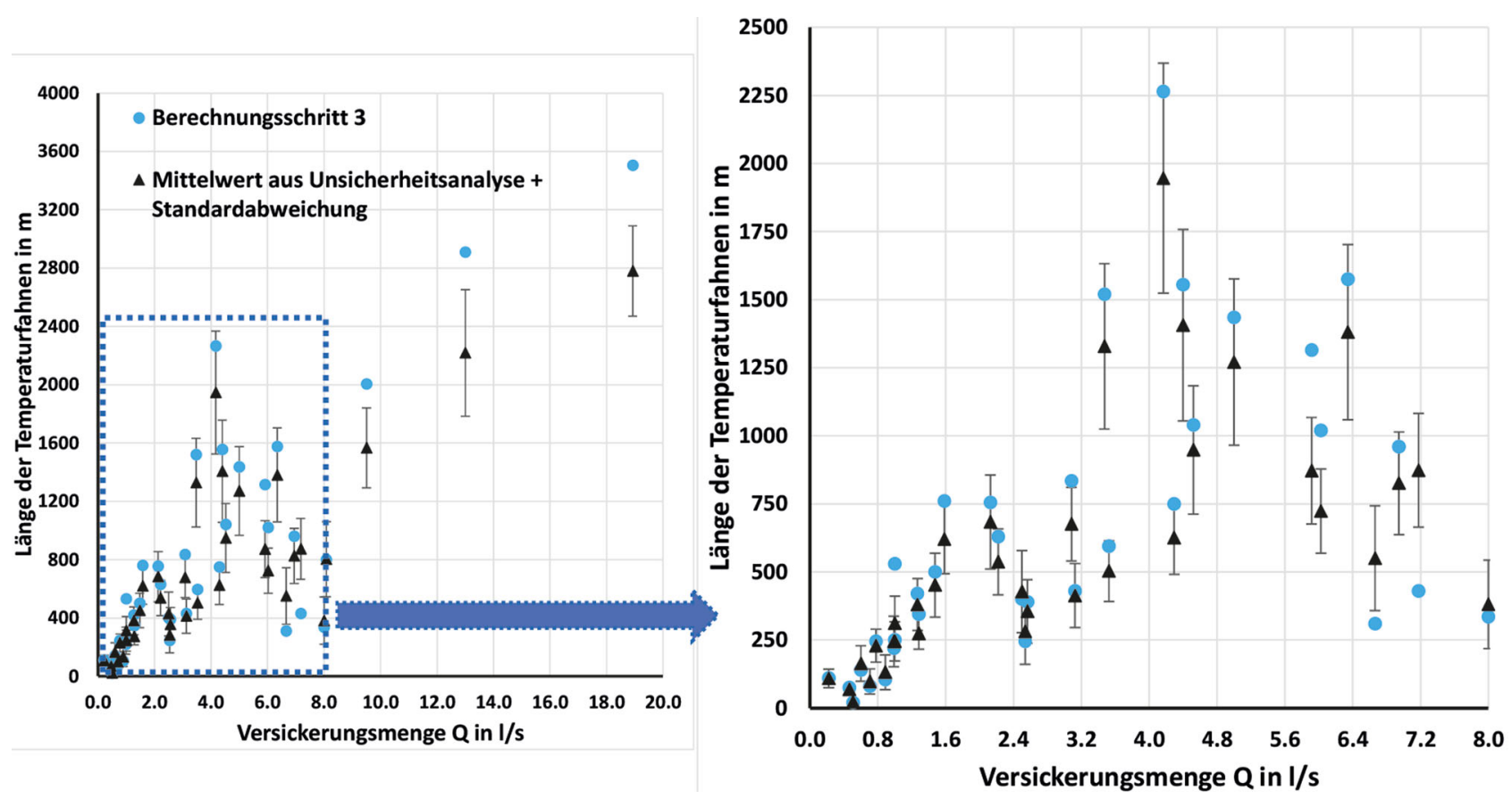

Abb. 7 Länge der Temperaturfahnen über Versickerungsmenge

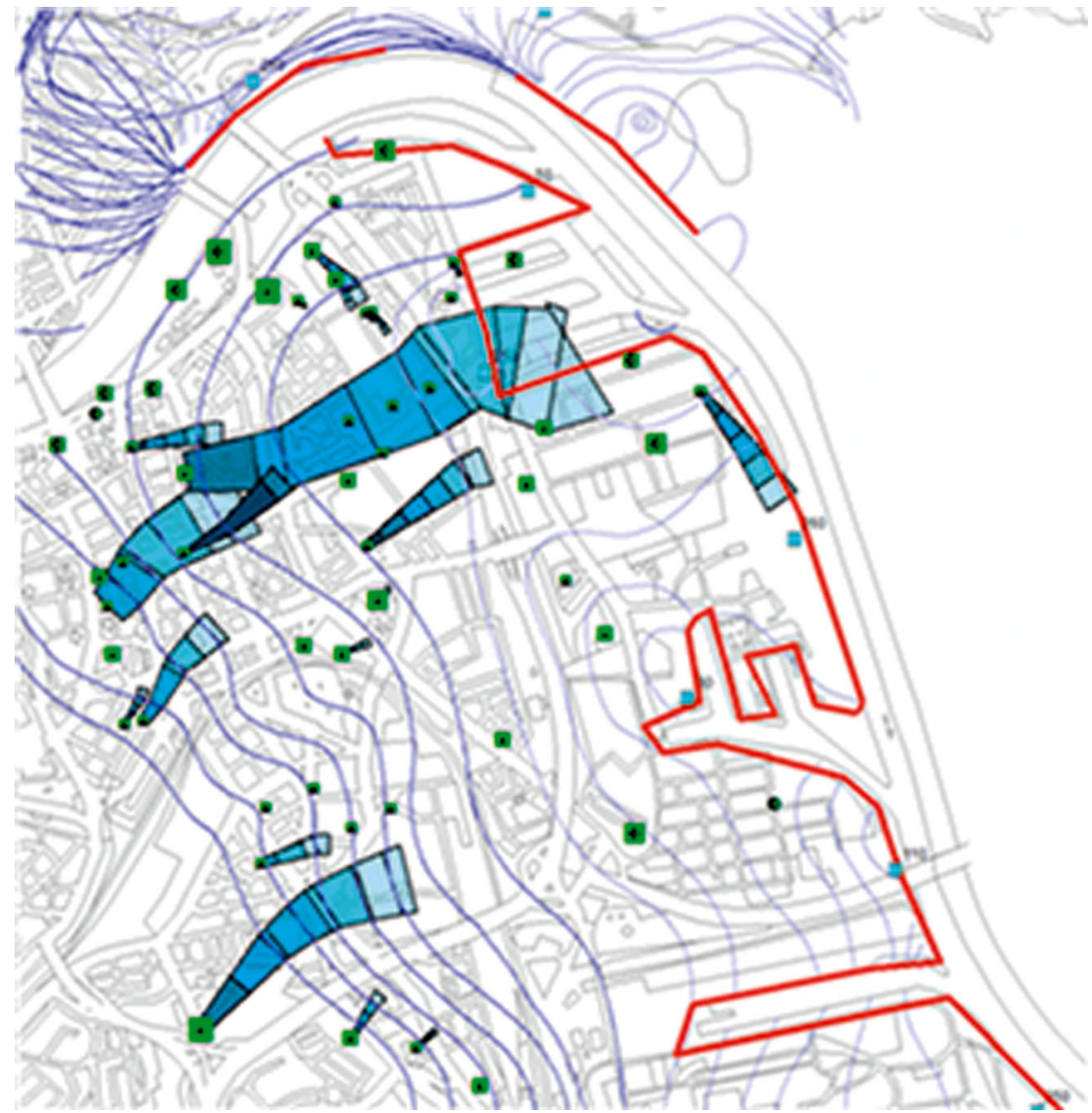

Abb. 8 Temperaturfahnen für die Grundwasserentnahmen 2012

\subsection{Ergebnisse der Nutzwertanalyse am Beispiel von Variante 1 und Variante 6}

Einen Auszug aus der Nutzwertanalyse zeigt die nachfolgende Tab. 3, in der die Ergebnisse der Fernkälteversorgungsvarianten 1 und 6 dargestellt sind.

Die wesentlichsten Unterschiede lassen sich bei den Bewertungskriterien „Einfluss auf die Grundwassertemperatur" und „Einfluss auf andere Grundwassernutzungen" erkennen. Andere Grundwassernutzer in diesem Zusammenhang sind z. B. Betreiber von Wärmepumpen sowie Trink- und Brauchwassernutzer.

Bei Variante 1 wurde davon ausgegangen, dass keine wirkliche Verbesserung der Grundwassertemperatur erfolgen würde, da der Grundwasserleiter durch die zentralen Fernkälteerzeugungsanlagen, die das erwärmte Grundwasser zurückversickern, thermisch massiv beeinflusst werden würde (siehe Abb. 10). Der Einfluss auf andere Grundwassernutzer wurde aus diesem Grund als sehr hoch angenommen.

Im Gegensatz dazu erfolgt durch die zentralen Erzeugeranlagen in Variante 6 kaum eine thermische Beeinflussung des Grundwassers (wenn, dann im Randbereich des Grundwasserleiters, in der Nähe der Dichtwände). Einige der Einzelanlagen verbleiben allerdings und hätten weiterhin einen nachteili- 


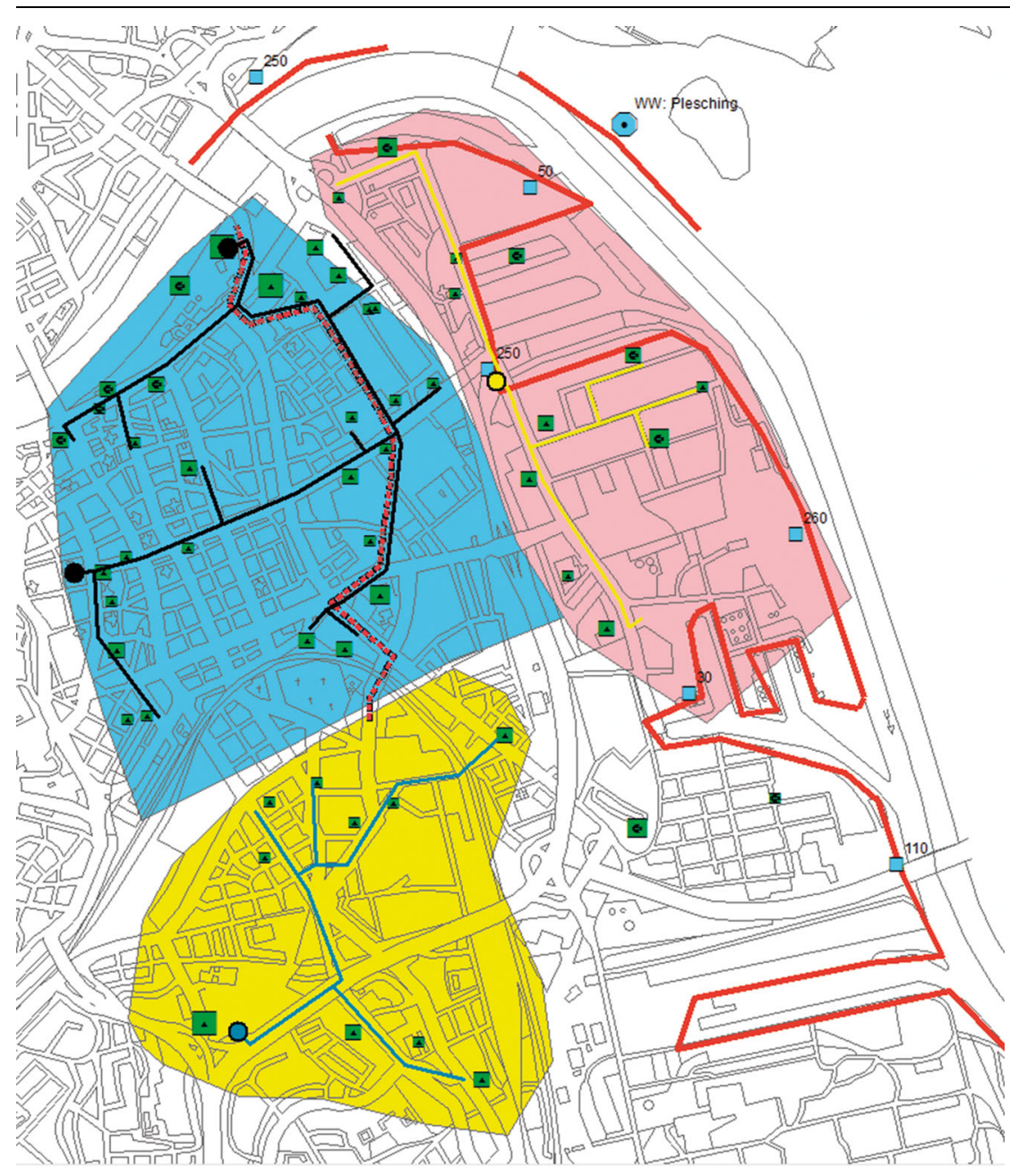

Abb. 9 Variante 1

gen thermischen Effekt. Im Vergleich zur Ausgangssituation stellt dies aber eine deutliche Verbesserung dar. Der Einfluss auf andere Grundwassernutzer würde sich ebenfalls im Vergleich zur Ausgangssituation verbessern, da die thermische Beeinflussung im nördlichen Teil des Bearbeitungsgebiets weg fallen würde.

\section{Zusammenfassung}

Die Grundwassernutzung für die Kälteerzeugung ist eine bereits praktizierte und umweltfreundliche Methode zur Kühlung von Gebäuden und technischen Anlagen. In Linz werden seit einigen Jahren entsprechende Anlagen betrieben. Die Grundwassernutzung zur Kälteerzeugung benötigt grundsätzlich eine wasserrechtliche Bewilligung und es ist darauf zu achten, dass das Grundwasser als wesentliche Ressource für die Trinkwassererzeugung nicht gefährdet wird.
In den von der DonauConsult Zottl \& Erber ZT GmbH $(2004,2006)$ durchgeführten Studien zur Grundwasserbewirtschaftung Linz (im Auftrag des Landes Oberösterreich) wurden erhöhte Grundwassertemperaturen festgestellt. Im Zuge der in diesem Artikel vorgestellten strategischen Studie (Fernkälte Linz - Modul I, Wasserwirtschaftliche Grundlagen 2013) wurde unter anderem ein möglicher thermischer Einfluss von Kältenutzungsanlagen auf die Grundwassertemperatur untersucht. Zudem wurden in der hier vorgestellten Studie mögliche Varianten zur Verhinderung allfälliger weiterer Temperaturbeeinflussungen sowie von möglichen zukünftigen Nutzungskonflikten entwickelt und bewertet.

Die im Bearbeitungsgebiet der Stadt Linz vergebenen Wasserrechte wurden eingangs ausgewertet. Es wurden insgesamt 54 Kältenutzer im Bearbeitungsgebiet lokalisiert. Für diese wurden auf Basis der jährlichen, durchschnittli- chen Konsensmengen Temperaturfahnen nach Ingerle (1988), modifiziert durch Rauch (1992), berechnet und in einem GIS-Programm (ARCMAP 10.1) grafisch dargestellt. Die trapezförmigen Temperaturfahnen wurden in einem zweiten Schritt an die Fließrichtung des Grundwasserleiters angepasst. In einem dritten Berechnungsschritt wurden zudem Durchlässigkeitsbeiwert und Grundwasserstandshöhengefälle über den Fließweg gemittelt und in die Berechnungen einbezogen. Für alle 3 Berechnungsschritte waren Bereiche zu erkennen, in denen sich die Temperaturfahnen stark überschneiden. Die Wahrscheinlichkeit einer Grundwassererwärmung in diesen Überschneidungsbereichen ist daher als erhöht anzusehen. Diese Überschneidungsbereiche wiesen gemäß der von DonauConsult Zottl \& Erber ZT GmbH erstellten Karten auf Basis von gemessenen maximalen Grundwassertemperaturen bereits 2006 erhöhte Grundwassertemperaturen auf.

Zusätzlich wurden die Kältenutzer hinsichtlich der 2012 tatsächlich versickerten Grundwassermengen angefragt. Mit den gemeldeten Versickerungsmengen wurden ebenfalls Temperaturfahnen berechnet. Die Größe der Temperaturanomalien war zwar im Vergleich zu den vorherigen Berechnungen deutlich geringer, aber auch hier waren Überschneidungen erkennbar.

Um die bereits erkennbare Grundwassererwärmung nicht weiter zu verschärfen und um mögliche zukünftige Nutzungskonflikte zu vermeiden wurden anschließend 7 Varianten für mögliche zentrale Fernkälteanlagen sowie möglicher Kältenetztrassierung definiert. Die Varianten wurden mittels Nutzwertanalyse auf Basis technischer, wirtschaftlicher sowie ökologischer Kriterien bewertet. Zwei dieser Varianten wurden in diesem Artikel näher vorgestellt. Dazu zählt auch Variante 6, die sich innerhalb der 7 Varianten auf Basis der Nutzwertanalyse als Vorzugsvariante herausgestellt hat. Es handelt sich dabei um eine semi-zentrale Variante. Dies bedeutet, dass nicht alle bestehenden Kältenutzer an das Fernkältenetz angeschlossen werden. Das vorgeschlagene Fernkältenetz weist eine Netzlänge von ca. $13,1 \mathrm{~km}$ auf und wird mit 3 Fernkältezentralen gespeist. Die Trasseneffizienz mit $1.114 \mathrm{~m}^{3} / \mathrm{m}$ entsprach dem zweithöchsten Anschlusswert. Die Vorteile dieser Variante sind neben der 


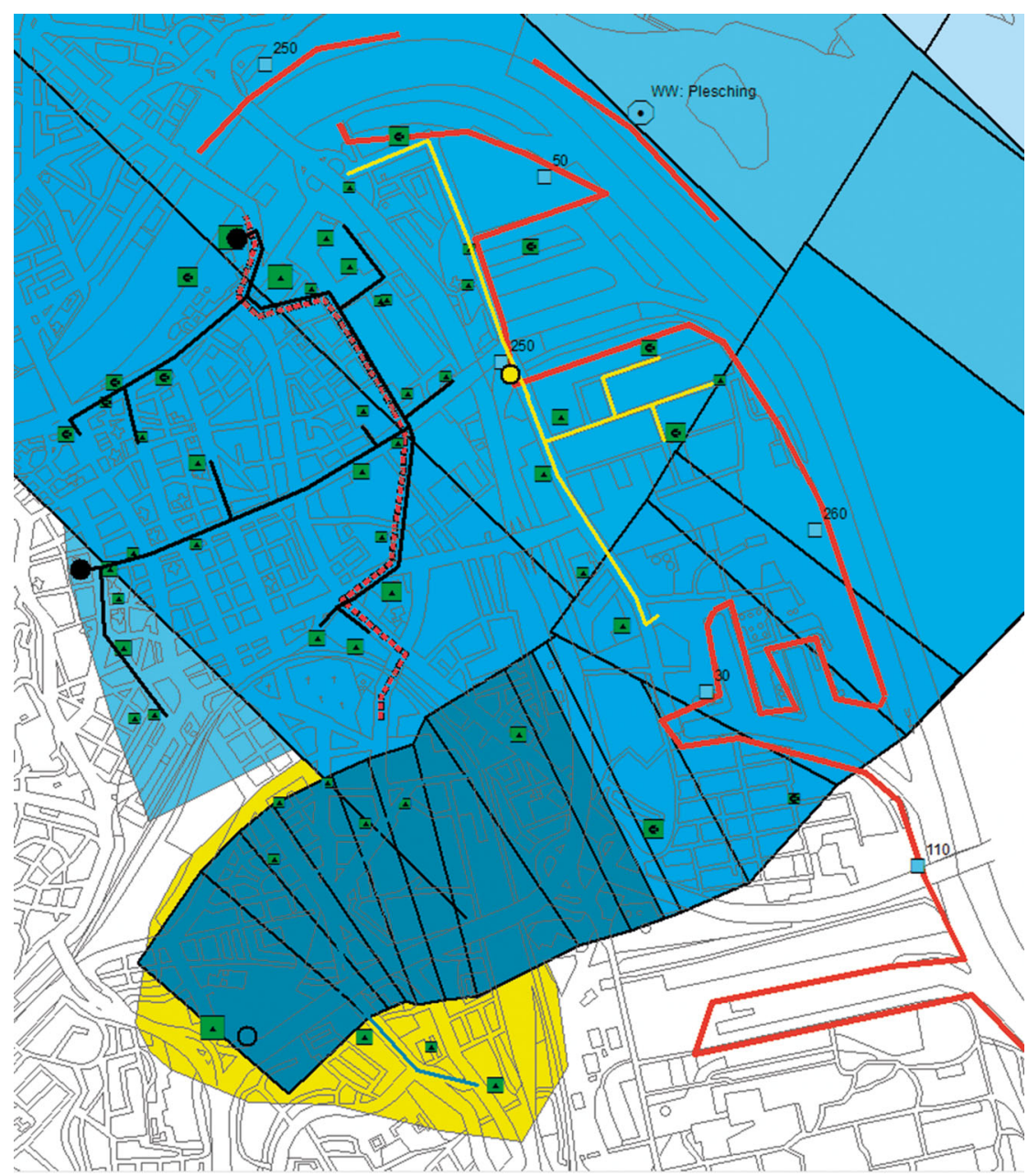

Abb. 10 Variante 1-Temperaturfahnen kompakten Trassenführung, die Nutzung bereits bestehender Anlagen zur Kälteerzeugung, wie z. B. die Fernkältezentrale der Linz AG. Zusätzlich führt diese Variante $\mathrm{zu}$ einem geringeren thermischen Grundwassereinfluss als vergleichbare andere Varianten.

Abschließend kann festhalten werden, dass die vereinfachte Thermalfahnenberechnung nach ÖWAV-Regelblatt 207 (2009) grundsätzlich für eine erste Abschätzung und Visualisierung des Temperatureinflusses von thermischen Nutzungsanlagen geeignet ist. Auch für die grobe Abschätzung wasserwirtschaftlicher Vor- und Nachteile möglicher Fernkältevarianten ist das Verfahren anwendbar. Die Bewertung des mit der gewählten Netzführung einhergehenden zukünftigen Wegfalls dezentraler Wärmequellen ist damit möglich. Ebenso die erste Bewertung möglicher Standorte für eine zentrale Kältegewinnung in Hinblick auf deren Einfluss auf die Veränderung der Grundwassertemperatur. Die Analyse der Ergebnisunsicherheit aufgrund der vorwiegend unsicheren Eingangsparameter dieses vereinfachten Berechnungsverfahrens wird daher empfohlen. Diese liefert transparente Ergebnisse in Hinblick auf mögliche Bandbreiten der Berechnungsergebnisse.

Für die Detailplanung von Fernkälteanlagen sollten in jedem Fall Untersuchungen auf Basis detaillierter Grundwassermodelle und eine Simulation unterschiedlicher Zukunftsszenarien, unter anderem in Hinblick auf den zukünftigen Kältebedarf, durchgeführt werden.

Open access funding provided by Graz University of Technology.

Open Access Dieser Artikel wird unter der Creative Commons Namensnennung 4.0 International Lizenz (http://creativecommons.org/licenses/ by/4.0/deed.de) veröffentlicht, welche die Nutzung, Vervielfältigung, Bearbeitung, Verbreitung und Wiedergabe in jeglichem Medium und Format erlaubt, sofern Sie den/die ursprünglichen $\mathrm{Au}$ tor(en) und die Quelle ordnungsgemäß nennen, einen Link zur Creative Commons Lizenz beifügen und angeben, $o b$ Änderungen vorgenommen wurden. 


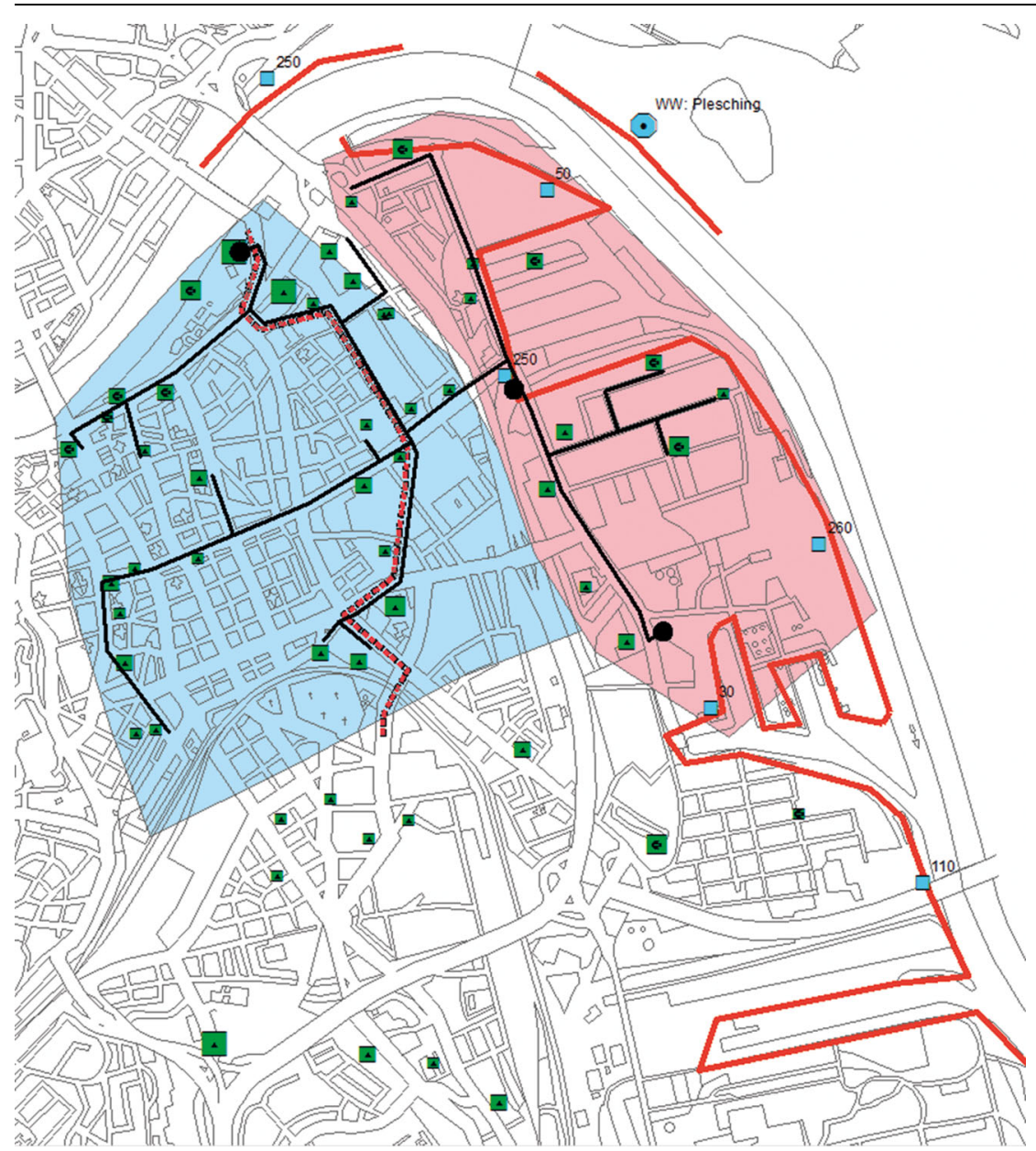

Abb. 11 Variante 6 


\section{Originalarbeit}

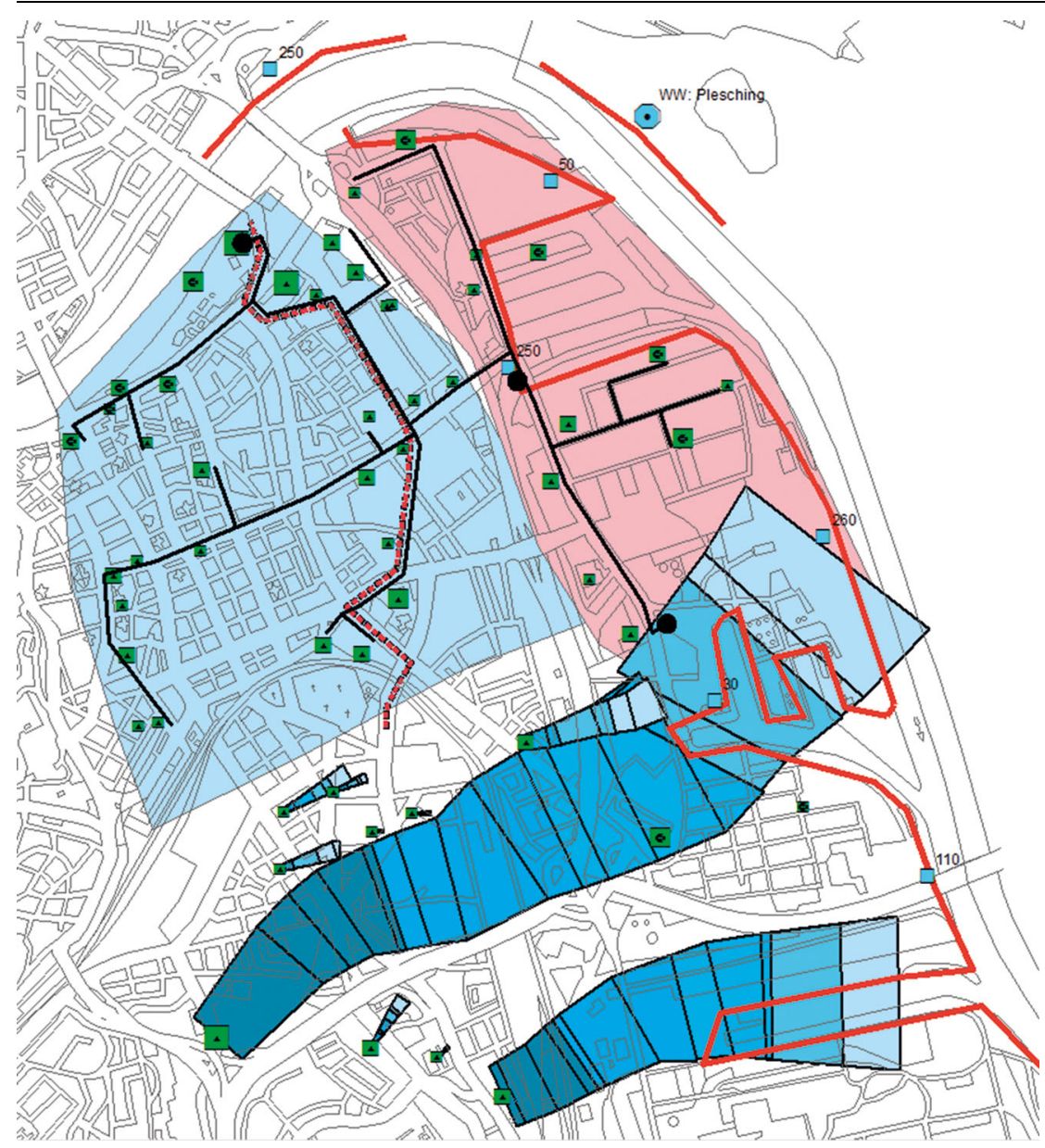

Abb. 12 Variante 6-Temperaturfahnen

Tab. 3 Auszüge aus der Nutzwertanalyse

\begin{tabular}{|c|c|c|c|c|c|}
\hline Wasserwirtschaft/ Umwelt & & $\begin{array}{l}\text { Variante } 1 \\
\text { ungew. }\end{array}$ & gew. & $\begin{array}{l}\text { Variante } 6 \\
\text { ungew. }\end{array}$ & gew. \\
\hline Einfluss auf den Grundwasserstand & $10 \%$ & 2 & 20 & 2 & 20 \\
\hline Einfluss auf die Grundwassertemperatur & $10 \%$ & 1 & 10 & 4 & 40 \\
\hline Verringerung der $\mathrm{CO}_{2}$-Emissionen & $5 \%$ & 4 & 20 & 4 & 20 \\
\hline Energiebedarf & $5 \%$ & 4 & 20 & 4 & 20 \\
\hline \multicolumn{6}{|l|}{ Wasserrechte/ Fremde Rechte } \\
\hline Konfliktpotential mit vorhandenen Grundwassernutzern & $10 \%$ & 1 & 10 & 2 & 20 \\
\hline Einfluss auf andere Grundwassernutzungen & $5 \%$ & 1 & 5 & 4 & 20 \\
\hline \multicolumn{6}{|c|}{ Beitrag zur Sicherung des Wirtschaftsstandortes } \\
\hline sichere Versorgung vorhandener Kältenutzer & $15 \%$ & 5 & 75 & 4 & 60 \\
\hline Stabilisierung und Verbesserung der Kühlleistung & $10 \%$ & 5 & 50 & 4 & 40 \\
\hline $\begin{array}{l}\text { Erweiterung von Kältenutzungen/ zusätzliche Kältenut- } \\
\text { zer }\end{array}$ & $10 \%$ & 4 & 40 & 5 & 50 \\
\hline \multicolumn{6}{|l|}{ Umsetzbarkeit } \\
\hline Synergie mit geplanter Straßenbahntrasse & $10 \%$ & 5 & 50 & 5 & 50 \\
\hline Trasseneffizienz & $10 \%$ & 4 & 40 & 5 & 50 \\
\hline Summe & $100 \%$ & 340 & & 390 & \\
\hline
\end{tabular}


Abwasseremissionsverordnung Kühlsysteme und Dampferzeuger i. d. F. vom 27. Mai 2003 Bundeskanzleramt, Rechtsinformationssystem (RIS), URL: http://www.ris.bka.gv.at

Bohne, D. (2014): Technischer Ausbau von Gebäuden und nachhaltige Gebäudetechnik, 10. aktualisierte Auflage, Springer Vieweg, Wiesbaden

Ingerle, K. (1988): Beitrag zur Berechnung der Abkühlung des Grundwasserkörpers durch Wärmepumpen, Österreichische Wasserwirtschaft, Jg. 40, H. 11/12, 1988

IPCC (2014): Climate Change 2014: Synthesis Report. Contribution of Working Groups I, II and III to the Fifth Assessment Report of the Intergovernmental Panel on Climate Change [Core Writing Team, R.K. Pachauri and L.A. Meyer (eds.)]. IPCC, Geneva, Switzerland, $151 \mathrm{pp}$
Krakow, S., Fuchs-Hanusch, D. (2013): Strategische Studie, Fernkälte Linz - Modul 1, Wasserwirtschaftliche Grundlagen, URL: http:// www2.land-oberoesterreich.gv.at, zuletzt abgerufen am 15.02.2016

Land Oberösterreich, DonauConsult Zottl \& Erber ZT GmbH (2004): Grundwasserbewirtschaftung Linz, Hydrologische und thermische IstSituation, Linz: Amt der Oö. Landesregierung Wasserwirtschaft, Grund- und Trinkwasserwirtschaft

Land Oberösterreich, DonauConsult Zottl \& Erber ZT GmbH (2006): Grundwasserbewirtschaftung Linz, Grundwassertemperatur - Messprogramm 2003 - 2005, Linz: Amt der Oö. Landesregierung, Wasserwirtschaft, Grund- und Trinkwasserwirtschaft

Rauch, W. (1992): Ausbreitung von Temperaturanomalien im Grundwasser, Dissertation,
Fakultät für Bauingenieurwesen, Universität Innsbruck, ISBN 3-900259-25-9, 1992

Sitzenfrei, R., Rauch, W. (2014): Anwendungsgrenzen einfacher analytischer Lösungen zur Bestimmung von Temperaturanomalien im Grundwasser. In: gwf - Wasser/ Abwasser, Band 155, Ausgabe 3, Seite 330-339

Wasserrechtsgesetz 1959 i. d. F. vom 1. August 2014: Bundeskanzleramt, Rechtsinformationssystem (RIS), URL: http://www.ris.bka.gv.at ÖWAV-Regelblatt 207 (2009): Thermische Nutzung des Grundwassers und des Untergrundes Heizen und Kühlen, 2., vollständig überarbeitete Auflage, Österreichischer Wasser- und Abfallwirtschaftsverband, Wien

ÖWAV-Arbeitsbehelf 43 (2014): Leitfaden zur Anwendung der Thermalfahnenformel des ÖWAV-Regelblattes 207, Österreichischer Wasser- und Abfallwirtschaftsverband, Wien 